\title{
Avaliação da qualificação em tuberculose para profissionais de saúde: proposta de um modelo sistematizado
}

\author{
Evaluation of tuberculosis qualification for healthcare professionals: proposal of a systematic \\ model
}

Evaluación de cualificación en tuberculosis para profesionales de la salud: propuesta de modelo sistemático

Leticia Auxiliadora Fragoso da Silva ORCID: https://orcid.org/0000-0003-3132-6030 Centro Universitário São Lucas, Brasil E-mail: letfragoso@hotmail.com

Ana Lúcia Escobar

ORCID: https://orcid.org/0000-0003-4386-0863 Universidade Federal de Rondônia, Brasil E-mail: ana@unir.br

Cleoni Alves Mendes de Lima ORCID: https://orcid.org/0000-0002-8453-0046 Laboratório Central de Saúde Pública de Rondônia, Brasil E-mail: cleoniml@yahoo.com.br

Reinaldo Antônio da Silva-Sobrinho ORCID: https://orcid.org/0000-0003-0421-4447 Universidade Estadual do Oeste do Paraná, Brasil E-mail: reisobrinho@yahoo.com.br

Melisane Regina Lima Ferreira ORCID: https://orcid.org/0000-0003-1694-5124 Universidade de São Paulo, Brasil E-mail: melisane1206@gmail.com

Nathalia Halax Orfão

ORCID: https://orcid.org/0000-0002-8734-3393 Universidade Federal de Rondônia, Brasil E-mail: nathaliahalax@hotmail.com

\begin{abstract}
Resumo
Objetivo: propor um modelo sistematizado de avaliação da qualificação em tuberculose (TB) para profissionais de saúde. Método: estudo de investigação metodológica quantitativa, desenvolvido no município de Porto Velho-RO, em três etapas para elaboração, validação de conteúdo e teste-piloto do instrumento de avaliação, para ser aplicado nas qualificações em TB, após atendidos os preceitos éticos. O primeiro instrumento (pré e pós-teste), foi constituído de 18 questões divididas em três seções. O segundo instrumento (pós-teste tardio), foi composto de duas questões abertas. A validação dos instrumentos ocorreu a partir da avaliação acerca da clareza, relevância e pertinência das questões por peritos na temática. Resultados: os instrumentos permaneceram com a mesma quantidade de itens, porém foram alterados quanto à escrita para melhor compreensão. Conclusões: o instrumento de avaliação elaborado possui validade de conteúdo e aparência, e é compatível para a avaliação da qualificação em TB, como ferramenta de feedback para gestor e participantes.
\end{abstract}

Palavras-chave: Tuberculose; Qualificação profissional em saúde; Pessoal de saúde; Estudos de validação.

\begin{abstract}
Objective: to propose a systematic model for the assessment of tuberculosis (TB) qualification for health professionals. Method: quantitative methodological investigation study, developed in the city of Porto Velho-RO, in three stages for the elaboration, content validation and pilot test of the evaluation instrument, to be applied in the qualifications in TB, after complying with the ethical precepts. The first instrument (pre and post-test), consisted of 18 questions divided into three sections. The second instrument (late post-test), was composed of two open questions. The validation of the instruments occurred based on the assessment of the clarity, relevance and pertinence of the questions by experts on the subject. Results: the instruments remained with the same number of items, but they were changed in terms of writing for better understanding. Conclusions: the evaluation instrument developed has content and appearance validity, and is compatible for the evaluation of TB qualification, as a feedback tool for managers and participants.
\end{abstract}

Keywords: Tuberculosis; Professional qualification in health; Health personnel; Validation studies. 


\begin{abstract}
Resumen
Objetivo: proponer un modelo sistemático para la evaluación de la calificación de tuberculosis (TB) para profesionales de la salud. Método: estudio de investigación metodológica cuantitativa, desarrollado en la ciudad de Porto Velho-RO, en tres etapas para la elaboración, validación de contenido y prueba piloto del instrumento de evaluación, para ser aplicado en las calificaciones en TB, luego de cumplir con los preceptos éticos. El primer instrumento (pre y post-test), constaba de 18 preguntas divididas en tres apartados. El segundo instrumento (posprueba tardía), estuvo compuesto por dos preguntas abiertas. La validación de los instrumentos se dio a partir de la valoración de la claridad, relevancia y pertinencia de las preguntas por parte de expertos en el tema. Resultados: los instrumentos se mantuvieron con el mismo número de ítems, pero se modificaron en cuanto a redacción para una mejor comprensión. Conclusiones: el instrumento de evaluación desarrollado tiene validez de contenido y apariencia, y es compatible para la evaluación de la calificación de TB, como herramienta de retroalimentación para gestores y participantes.
\end{abstract}

Palabras clave: Tuberculosis; Cualificación profesional en salud; Personal de salud; Estudios de validación.

\title{
1. Introdução
}

A tuberculose (TB) se constitui um problema tão antigo quanto atual e parece não sair de evidência. No ano de 2018 , 10 milhões de pessoas adoeceram e destas, 1,2 milhões chegaram à óbito pela doença. Já no Brasil, no mesmo ano, mais de 95 mil novos casos de TB foram notificados, ocasionando 4,8 mil óbitos pela doença, configurando-a como a primeira entre as principais causas de morte por agentes infecciosos, superando o HIV/aids e a malária (World Health Organization, 2019).

Por ser uma doença tratável e curável com medicamentos de baixo custo, acredita-se que todo esforço na qualificação de processos que culminem em sua erradicação e controle são essenciais (Lima et al., 2016), tais como a melhoria da capacidade técnica dos profissionais de saúde e o empoderamento de concepções e práticas pertinentes às suas competências e habilidades.

Em face à diversidade da assistência que deve ser ofertada aos doentes de TB, faz-se necessária a articulação entre a interprofissionalidade e a qualificação das diferentes categorias profissionais que visam intensificar as ações de promoção, prevenção e recuperação da saúde para o doente de TB, família e comunidade. Esta qualificação da atenção à saúde perpassa diferentes dimensões estruturais, organizacionais e aspectos interprofissionais e de educação em saúde (Reeves, 2016).

A Educação Permanente em Saúde (EPS) propõe as transformações do trabalho para que o setor de saúde seja um lugar para atuação crítica, reflexiva, propositiva, compromissada e tecnicamente competente (Ceccim, 2005). A qualificação, como parte dessa política, é definida como o esforço das organizações para garantir o aperfeiçoamento dos profissionais em suas deficiências e prepará-los para novas funções, bem como adaptá-los às novas tecnologias (Borges-Andrade, 2006). Assim, os treinamentos e qualificações centrados na transmissão de conhecimento são superados pela problematização e autoanálise da rotina cotidiana de trabalho (Borges-Andrade, 2006; Yost et al., 2014).

Neste sentido, a assistência prestada ao doente de TB não depende somente das competências e habilidades, mas também da avaliação da aprendizagem. Para cada qualificação, deve-se utilizar a melhor forma de certificar e mensurar o progresso de quem está sendo avaliado, verificar seu desempenho e recomendar os reajustes complementares. A qualificação em TB é um espaço que poderia proporcionar discussões com contextos de problematização em que o participante mobiliza transversalmente as funções de planejamento, gestão, execução, monitoramento e avaliação, além de contribuir para o fortalecimento da definição do trabalho em equipe.

A utilização de instrumentos de avaliação pode gerar boas medidas, de maneira a garantir que todos os aspectos das ações sejam discutidos, assim como as evidências e critérios necessários que devem ser considerados em atividades específicas, como na TB. Em relação aos instrumentos de investigação, os questionários representam um dos artifícios mais relevantes para aquisição de informações. No entanto, é importante que esses instrumentos apresentem qualidade e garantia da confiabilidade dos itens avaliados, visando garantir a precisão para mensurar o que propõe (Silva et al., 2015).

Apesar da relevância da temática, poucos estudos focam a avaliação da qualificação em TB, restringindo-se apenas às estratégias utilizadas para detecção de casos, busca de sintomáticos respiratórios, controle de contatos, métodos diagnósticos, 
indicadores de resultados e avaliação de serviços. Desta forma, este estudo teve como objetivo propor um modelo sistematizado de avaliação da qualificação em TB para profissionais de saúde.

\section{Metodologia}

Trata-se de um estudo de investigação metodológica quantitativa, realizada no município de Porto Velho, capital do estado de Rondônia, selecionado pela Coordenação Estadual do Programa de Controle da TB (PCT) para as qualificações, desde 1997, por meio de projetos de treinamentos designados “Capacitação Básica em TB", com o objetivo principal de qualificar os profissionais de saúde para assumirem as ações de controle e vigilância em TB.

As qualificações em TB tinham duração de 24 horas, sendo essas distribuídas em oito horas, durante três dias consecutivos, para um público-alvo médio de 60 pessoas, distribuído entre os horários matutinos e vespertinos. Tal atividade era oferecida duas vezes ao ano, mediante convocação da participação mínima de um representante por categoria profissional de cada unidade. As temáticas selecionadas para a qualificação eram embasadas nos manuais do Ministério da Saúde (Brasil, 2011; 2018), e ministradas de forma expositiva por médicos e enfermeiros que atuavam nos serviços de TB, bem como convidados da coordenação, levando em consideração o conhecimento, vivência prática e envolvimento dos profissionais componentes na temática abordada.

Para contemplar o objetivo deste estudo, realizou-se três etapas: 1- Elaboração do instrumento de avaliação com base nos princípios do Programa Nacional de Controle da TB (PNCT) (Brasil, 2011; 2018); 2- Validação de conteúdo do instrumento por peritos (Polit et al., 2009) que possuíssem três anos de experiência na temática; e 3- Teste-piloto para aplicação do instrumento com os profissionais de saúde, convocados para a qualificação em TB, no primeiro semestre de 2017.

Como critérios de inclusão, foram considerados os profissionais que trabalhavam na assistência ao doente de TB, convocados para participar da qualificação e que estiveram presentes no evento. Foram excluídos, aqueles que estiveram de licença e/ou férias, bem como os que se ausentaram em pelo menos uma das etapas da qualificação em TB. Os dados foram digitados, armazenados e analisados por meio de estatística descritiva, com intuito de avaliar o instrumento e, de modo complementar, analisar o conhecimento adquirido pelos profissionais de saúde e a aplicabilidade da qualificação na prática profissional.

Atendendo às recomendações contidas na resolução nº 466, de 12 de dezembro de 2012, do Conselho Nacional de Saúde e das diretrizes e normas regulamentadoras de pesquisa envolvendo seres humanos (Brasil, 2012), este projeto foi submetido e aprovado pelo Comitê de Ética em Pesquisa (CEP) da Universidade Federal de Rondônia (UNIR), conforme parecer consubstanciado número 1.849.728 CEP/NUSAU/UNIR. Ressalta-se que o teste-piloto foi realizado mediante assinatura do Termo de Consentimento Livre e Esclarecido.

\section{Resultados}

O primeiro instrumento incluiu o pré e pós-teste, versando sobre os mesmos tópicos e idêntico grau de dificuldade, constituído por 18 questões divididas em três seções, sendo elas: características sociodemográficas, qualificação prévia em TB e conhecimento sobre TB. O segundo instrumento (pós-teste tardio) foi aplicado 60 dias após a qualificação em TB para verificar a sua aplicabilidade na prática profissional, sendo este constituído de duas questões abertas (Tabela 1). 
Tabela 1. Caracterização das etapas dos instrumentos aplicados, segundo suas seções e variáveis, para avaliação da qualificação em tuberculose para profissionais de saúde, Porto Velho, Rondônia, 2017.

\begin{tabular}{|c|c|c|}
\hline Etapas & Seção & Variáveis \\
\hline \multirow{24}{*}{$\begin{array}{c}\text { Pré e Pós- } \\
\text { teste }\end{array}$} & \multirow{6}{*}{ I. Características sociodemográficas } & Escolaridade \\
\hline & & Titulação acadêmica \\
\hline & & Categoria profissional \\
\hline & & Ano de formação \\
\hline & & Local de trabalho \\
\hline & & Tempo de atuação neste local de trabalho \\
\hline & \multirow{6}{*}{ II. Qualificação prévia em TB } & Participação em alguma qualificação em TB \\
\hline & & Quem ofereceu a qualificação em TB \\
\hline & & Número de qualificações em TB que participou \\
\hline & & Ano da última qualificação em TB \\
\hline & & Acompanhou algum caso de TB \\
\hline & & Se acompanhou foi como interventor ou observador \\
\hline & \multirow{12}{*}{ III. Conhecimento sobre TB } & Condução de um caso suspeito de TB desde o diagnóstico até o término do \\
\hline & & tratamento no sistema de saúde \\
\hline & & Entendimento sobre sintomático respiratório \\
\hline & & Existência de grupos de maior vulnerabilidade/ risco para desenvolver TB \\
\hline & & Exames utilizados para o diagnóstico da TB \\
\hline & & Como deve ocorrer o tratamento da TB \\
\hline & & Como deve ocorrer o Tratamento Diretamente Observado (TDO) \\
\hline & & Entendimento sobre Infecção Latente de TB (ILTB) \\
\hline & & Como deve ocorrer o diagnóstico e tratamento da ILTB \\
\hline & & Como deve ocorrer o acompanhamento das ações de vigilância para o \\
\hline & & controle da TB \\
\hline & & Situação de encerramento de um caso de TB \\
\hline Pós-teste & IV. Aplicabilidade das ações de TB na & Está desenvolvendo ações de controle da TB \\
\hline tardio & prática profissional & Está encontrando dificuldades para realizar as ações de controle da TB \\
\hline
\end{tabular}

Legenda: TB: Tuberculose, TDO: Tratamento Diretamente Observado, ILTB: Infecção Latente da Tuberculose. Fonte: Elaborada pelos autores.

Os critérios utilizados para construir tais instrumentos foram sua aplicabilidade e recomendações atuais, em conformidade com os padrões nacionais e internacionais para o controle da TB. A linguagem empregada foi dirigida para o entendimento simples, claro e objetivo, sem dubiedade na interpretação. Em se tratando do estilo empregado no instrumento, buscou-se torná-lo compreensível e, por conseguinte, fácil de ser respondido.

A validação dos instrumentos ocorreu a partir da avaliação acerca da clareza, relevância e pertinência das questões por 11 peritos na temática, dos 15 convidados, os quais foram contatados por meio de correspondência via correio eletrônico, explicitando o objetivo do estudo e estipulando o prazo de até 15 dias para retorno e efetivação do processo de avaliação. Os instrumentos permaneceram com a mesma quantidade de itens, porém, foram alterados quanto à escrita para melhor compreensão (Tabela 2). 
Tabela 2. Caracterização dos itens do instrumento validado, antes, com as sugestões e depois, para avaliação da qualificação em tuberculose para profissionais de saúde, Porto Velho, Rondônia, 2017.

\begin{tabular}{|c|c|c|c|}
\hline $\begin{array}{c}\text { Item do } \\
\text { Instrumento }\end{array}$ & Antes & Sugestões & Depois \\
\hline Objetivo & $\begin{array}{l}\text { Propor um modelo sistematizado de } \\
\text { avaliação para qualificação em } \\
\text { tuberculose }\end{array}$ & $\begin{array}{l}\text { Avaliar quem, agentes } \\
\text { atuantes, população ou } \\
\text { ambos? }\end{array}$ & $\begin{array}{l}\text { Propor um modelo sistematizado de } \\
\text { avaliação para qualificação em } \\
\text { tuberculose para profissionais de saúde. }\end{array}$ \\
\hline Qualificação & Qualificação & $\begin{array}{c}\text { Capacitação } \\
\text { Aperfeiçoamento } \\
\text { Sensibilização } \\
\text { Atividade de formação }\end{array}$ & Qualificação \\
\hline $\begin{array}{l}\text { Categoria } \\
\text { profissional }\end{array}$ & $\begin{array}{c}\text { Médico, Enfermeiro, Tec. } \\
\text { Enfermagem, Odontólogo, ACS, } \\
\text { Outros. }\end{array}$ & $\begin{array}{l}\text { Categorias estratificadas por } \\
\text { nível de competência }\end{array}$ & $\begin{array}{c}\text { ACS, Aux/Téc. enfermagem, } \\
\text { Enfermeiro, Médico, Odontólogo, } \\
\text { Outros. }\end{array}$ \\
\hline $\begin{array}{l}\text { Qualificação prévia } \\
\text { em TB, quem } \\
\text { ofereceu? }\end{array}$ & Pergunta aberta & $\begin{array}{l}\text { As ações de TB ocorrem em } \\
\text { ações bipartite, tripartite, } \\
\text { com academia também? }\end{array}$ & $\begin{array}{l}\text { Programa de Controle da Tuberculose } \\
\qquad \begin{array}{c}\text { (PCT) Município, } \\
\text { PCT Estado } \\
\text { PNCT } \\
\text { Outros }\end{array}\end{array}$ \\
\hline $\begin{array}{c}\text { Sintomático } \\
\text { respiratório (SR) }\end{array}$ & $\begin{array}{l}\text { Como deve ser o fluxo de atendimento } \\
\text { de um caso suspeito de TB desde o } \\
\text { diagnóstico da doença até o término do } \\
\text { tratamento no sistema de saúde do seu } \\
\text { município? }\end{array}$ & $\begin{array}{l}\text { Como você acha que deveria } \\
\text { ser o fluxo ou como deveria } \\
\text { ser? }\end{array}$ & $\begin{array}{l}\text { Quando um suspeito de TB é } \\
\text { identificado, como é feita a condução do } \\
\text { caso dentro dos serviços de saúde no seu } \\
\text { município, desde o diagnóstico até o } \\
\text { término do tratamento? }\end{array}$ \\
\hline
\end{tabular}

Legenda: TB: Tuberculose, ACS: Agente Comunitário de Saúde, PCT: Programa de Controle da Tuberculose, PNCT: Programa Nacional de Controle da Tuberculose.

Fonte: Elaborada pelos autores.

As aplicações do pré e pós-teste ocorreram de forma coletiva, com a participação dos profissionais de saúde, 30 minutos antes e após a qualificação, respectivamente, com a presença da pesquisadora, no período de nove a 11 de maio de 2017 . Essa qualificação foi ofertada pela Coordenação Estadual do PCT-RO, no período vespertino, totalizando excepcionalmente 12 horas, a qual contou com uma equipe de facilitadores, responsáveis pela condução do conteúdo programático, utilizando metodologia expositiva.

Ressalta-se que 69 pessoas participaram da referida qualificação, sendo que 39 foram excluídas, das quais 19 eram estudantes de graduação em enfermagem e 20 se ausentaram em pelo menos uma das etapas da qualificação em TB. Nesse sentido, participaram deste estudo 30 profissionais de saúde, sendo que a maioria tinha ensino superior completo (83\%), eram médicos (47\%), possuíam especialização (43\%), tempo de formação acadêmica de seis a dez anos (37\%), com atuação na Estratégia Saúde da Família (52\%) de um a cinco anos (37\%). Ressalta-se que 33\% dos participantes não responderam sobre qual era a sua unidade de lotação (Tabela 3). 
Tabela 3. Distribuição dos profissionais de saúde que participaram da capacitação em tuberculose $(\mathrm{n}=30)$, segundo características sociodemográficas, Porto Velho, Rondônia, 2017.

\begin{tabular}{|c|c|c|}
\hline Características Sociodemográficas & Variáveis & $\mathbf{N}(\%)$ \\
\hline \multirow{3}{*}{ Escolaridade } & Ensino médio & $4(4)$ \\
\hline & Ensino superior incompleto & $1(3)$ \\
\hline & Ensino superior completo & $25(83)$ \\
\hline \multirow{4}{*}{ Titulação acadêmica } & Especialização & $13(43)$ \\
\hline & Graduação & $11(37)$ \\
\hline & Não se aplica & $5(17)$ \\
\hline & Não respondeu & $1(3)$ \\
\hline \multirow{3}{*}{ Categoria profissional } & Médico & $14(47)$ \\
\hline & Enfermeiro & $10(33)$ \\
\hline & Técnico de enfermagem & $6(20)$ \\
\hline \multirow{5}{*}{ Tempo de formação acadêmica } & 1 a 5 & $9(30)$ \\
\hline & 6 a 10 & $11(37)$ \\
\hline & 11 a 20 & $5(17)$ \\
\hline & 21 a 28 & $2(7)$ \\
\hline & Não respondeu & $3(10)$ \\
\hline \multirow{6}{*}{ Unidade de lotação } & Unidade de Saúde da Família (USF) & $15(52)$ \\
\hline & Unidade Básica de Saúde (UBS) & $2(7)$ \\
\hline & Centro de Especialidades Médicas (CEM) & $1(3)$ \\
\hline & Distrito Sanitário Especial Indígena (DSEI) & $1(3)$ \\
\hline & Não respondeu & $10(33)$ \\
\hline & Ilegível & $1(3)$ \\
\hline \multirow{5}{*}{ Tempo de atuação na unidade de saúde } & Menos de um ano & $6(20)$ \\
\hline & 1 a 5 & $11(37)$ \\
\hline & 6 a 10 & $4(13)$ \\
\hline & Não respondeu & $9(30)$ \\
\hline & TOTAL & $30(100)$ \\
\hline
\end{tabular}

Legenda: Unidades de Saúde da Atenção Primária (USF: Unidade de Saúde da Família ou UBS: Unidade Básica de Saúde), CEM: Centro de Especialidades Médicas, DSEI: Distrito Sanitário Especial Indígena.

Fonte: Elaborada pelos autores.

No tocante à qualificação prévia em TB, verificou-se que a maioria não havia participado anteriormente (57\%). Entretanto, dentre aqueles que já haviam participado, $47 \%$ receberam a qualificação ofertada pelo PCT do município, pelo menos uma vez (26\%), há dois anos (14\%), sendo que um participante referiu que a última qualificação foi há 15 anos. Além disso, $73 \%$ já acompanharam algum caso de TB e atuaram como responsáveis por este (48\%) (Tabela 4). 
Tabela 4. Distribuição dos profissionais de saúde que participaram da qualificação em tuberculose $(\mathrm{n}=30)$, de acordo com a qualificação prévia em tuberculose, Porto Velho, Rondônia, 2017.

\begin{tabular}{|c|c|c|}
\hline Qualificação prévia em TB & Variáveis & $\mathbf{N}(\%)$ \\
\hline \multirow{2}{*}{ Participação em qualificação em TB } & Sim & $13(43)$ \\
\hline & Não & $17(57)$ \\
\hline \multirow{4}{*}{ Quem ofereceu a qualificação } & Programa TB município & $6(47)$ \\
\hline & Programa TB Estado & $2(15)$ \\
\hline & Outros & $3(23)$ \\
\hline & Não respondeu & $2(15)$ \\
\hline \multirow{3}{*}{ Número de qualificações que participaram } & 1 & $8(26)$ \\
\hline & 2 & $5(17)$ \\
\hline & Não se aplica & $17(57)$ \\
\hline \multirow{9}{*}{ Tempo da última qualificação (em anos) } & 1 & $1(3)$ \\
\hline & 2 & $4(14)$ \\
\hline & 3 & $2(7)$ \\
\hline & 5 & $1(3)$ \\
\hline & 15 & $1(3)$ \\
\hline & Ilegível & $1(3)$ \\
\hline & Não lembra & $1(3)$ \\
\hline & Não respondeu & $2(7)$ \\
\hline & Não se aplica & $17(57)$ \\
\hline \multirow{3}{*}{ Acompanhou algum caso de TB } & Sim & $22(73)$ \\
\hline & Não & $7(24)$ \\
\hline & Não respondeu & $1(3)$ \\
\hline \multirow{5}{*}{ Como acompanhou os casos* } & Responsável pelo caso & $13(48)$ \\
\hline & Agente observador & $6(22)$ \\
\hline & Não se aplica & $7(26)$ \\
\hline & Não respondeu & $1(4)$ \\
\hline & TOTAL & $30(100)$ \\
\hline
\end{tabular}

Legenda: TB: Tuberculose, PCT: Programa de Controle da Tuberculose.

*Alguns participantes responderam que acompanharam os casos de TB como responsável e observador concomitantemente.

Fonte: Elaborada pelos autores.

O conhecimento sobre a TB, pelos participantes, apresentou fragilidades na abordagem da condução de um caso suspeito dentro dos serviços de saúde, desde o diagnóstico até o término do tratamento, com respostas inadequadas no pré e pós-teste, sendo acrescentado apenas outros níveis de atenção.

Em relação ao entendimento sobre o que é um SR, observou-se, no pré e pós-teste, que a maioria relatou como sendo a pessoa com tosse há mais de três semanas, porém, existe equívocos sobre os sinais e sintomas da TB. Já no que diz respeito ao grupo de vulnerabilidade/risco para desenvolver TB, os profissionais conheciam a existência desse no pré-teste e inseriram outros grupos no pós-teste.

Quanto aos exames, os profissionais sabiam da existência e reconheciam o Teste Molecular Rápido para a TB (TMRTB) para o diagnóstico da doença, embora outros exames tenham sido relatados de forma equivocada no pré-teste, tais como VDRL e ressonância magnética. No pós-teste, foram verificadas fragilidades quanto ao momento de solicitação dos exames preconizados para o diagnóstico oportuno da TB. 
No que concerne à maneira como deveria ocorrer o tratamento da TB, três profissionais não responderam ao pré-teste. De modo geral, conheciam o tempo de duração do tratamento, nome dos medicamentos e o esquema terapêutico, ainda que tenha ocorrido os mesmos erros nas respostas, limitando-o ao TDO, cuja compreensão do conceito se restringe apenas à ingesta medicamentosa, de "segunda a sexta-feira".

Os profissionais compreenderam o significado da sigla ILTB (Infecção Latente da TB) no pré e pós-teste, no entanto não ficou claro os exames para diagnóstico, tempo de tratamento, critérios, grupos prioritários, investigação de contatos e acompanhamento das ações de vigilância para o controle da TB.

Quanto às situações de encerramento de um caso de TB, foram evidenciadas respostas equivocadas no que diz respeito a "exames", "tempo de tratamento (quatro meses)", "recuperação" e "baciloscopia de escarro para controle como forma/situação de encerramento".

No que concerne ao pós-teste tardio, este foi enviado via correio eletrônico aos participantes, dos quais apenas 50\% responderam. Com relação ao desenvolvimento das ações de TB, os participantes referiram à busca ativa de SR e faltosos, solicitação de TMR-TB e anotações no livro de registro de SR, bem como a notificação dos casos e acompanhamento contínuo com a participação da equipe multiprofissional. Outros complementaram com a vigilância dos contatos.

Quanto às dificuldades para realizar as ações de controle da TB, três não responderam ao questionamento e outros ressaltaram a falta eventual de insumos, deficiência na estrutura física da unidade de saúde, falta de auxílio para a pessoa de baixa renda, tais como vale-transporte e cesta básica, bem como a demora dos resultados de raio-X com laudo.

\section{Discussão}

Na qualificação em TB, os objetivos da aprendizagem, quando atingidos, podem se relacionar à aquisição de novos conhecimentos, habilidades e atitudes que podem refletir em mudanças a partir das competências treinadas (Abbad et al., 2013), uma vez que se caracteriza como um processo de aprendizagem e, a EPS é um recurso com vistas à melhoria da qualidade da assistência prestada à comunidade (Duarte et al., 2011). Todavia, para obter resultados consistentes, é preciso investigar algum tempo depois ou logo após a qualificação, a operacionalização do que foi aprendido na prática profissional (Noe et al. 2014), e não apenas a assimilação do conteúdo ensinado.

Por isso, tais objetivos devem ser delineados ainda na etapa do planejamento da qualificação e considerados como metas a serem alcançadas ao final do evento. Quando a qualificação é desenhada dessa maneira, é possível elaborar um instrumento de avaliação, de acordo com os objetivos propostos, e, idealmente, subsidiados por uma taxonomia de resultados de aprendizagem (Queiroga et al., 2012). A avaliação deve criar oportunidade de aprendizado diversificado, participativo, não hierarquizado, interdisciplinar, prospectivo e democrático (Brasil, 2015).

A qualificação em TB torna-se satisfatória quando é sustentada por propostas educativas capazes de desenvolver práticas e reflexões sobre o processo de trabalho, alicerçado na aprendizagem significativa para que não ocorra apenas a retenção da estrutura do conhecimento, mas que permita desenvolver a capacidade de transferir esse conhecimento para a sua possível utilização em contextos diferentes daquele em que ela se concretizou (Tavares, 2010). Neste sentido, é interessante que, durante a qualificação do profissional, seja adotada a problematização (Abbad et al., 2013) para estimular o pensamento crítico e a revisão de valores e práticas, assim como encontrar alternativas de solução e decisão (Zoboli \& Soares, 2012).

No que concerne ao tempo de atuação na unidade de saúde, este pode contribuir para que se desenvolvam ações mais resolutivas, favorecendo o diagnóstico precoce e melhorias no processo de trabalho. Assim, os esforços precisam ser intensificados com a EPS pelos gestores, a fim de que tragam benefícios em larga escala, além de ampliar a visão dos profissionais, emitindo melhor compreensão do controle da doença e de suas singularidades (Moreira et al., 2017). 
O controle da TB requer a garantia de acesso aos serviços de saúde e a disponibilidade de recursos humanos qualificados, cujo processo de trabalho inclui a incorporação de conhecimento para organizar a oferta e prestação do cuidado, desde a suspeita e manejo clínico até o encerramento do caso. Nestes termos, uma reduzida apropriação prévia de conteúdos essenciais, no que diz respeito ao adoecimento, poderia acarretar dificuldades para o desenvolvimento de medidas de controle, principalmente para a condução e manejo clínico de casos de TB (Duarte et al., 2011; Mariano et al., 2015).

Por meio de tais fragilidades, na qual não foi possível mensurar a diferença entre o momento do pré e pós-teste, entendese que um grupo permaneceu inseguro, ressaltando-se a importância da instrumentalização da avaliação da qualificação, com intuito de melhorar a assistência e estimular a reflexão acerca da efetiva aprendizagem por meio da problematização dos conteúdos e participação dos profissionais para a aplicabilidade em diferentes situações do cotidiano (Mariano et al., 2015).

\section{Considerações Finais}

Na comparação dos resultados do pré e pós-teste imediato e tardio, fica evidente que há retenção e aumento do conhecimento por parte dos profissionais em alguns tópicos, o que permite identificar o nível de eficácia dos conteúdos propostos, bem como sua contribuição para o desenvolvimento da aprendizagem. Por outro lado, outros itens não estavam coerentes, nos quais os participantes demonstraram ter pouca informação.

A partir dos resultados obtidos, considerou-se que o instrumento de avaliação elaborado possui validade de conteúdo e de aparência e, no momento, é compatível com o fim a que se propõe, qual seja avaliar a qualificação em TB para profissionais de saúde. Sugere-se pesquisas futuras para outros tipos de validação e tratamento dos dados, além de padronização da responsividade e do incentivo à aplicação, como instrumento de feedback para gestor e participantes, sabendo-se que somente a avaliação do aprendizado não garante obtenção total da eficácia de uma qualificação.

Entretanto, a característica do processo educativo em foco, com curto intervalo de tempo para discussão de temas referentes ao conhecimento sobre TB e metodologia expositiva predominante, deve ser apontada como fator inequivocamente limitante para a apreciação mais fidedigna dos efeitos no incremento cognitivo dos participantes para o aprofundamento de alguns conceitos que não obtiveram resultado expressivo em termos de assimilação, retenção, transferência e impacto.

\section{Agradecimentos}

Agradecemos à Coordenação Estadual do Programa de Controle da TB, ao Grupo de Estudos em TB (GET) da Universidade Federal de Rondônia, e aos profissionais de saúde que participaram e permitiram a realização deste estudo.

\section{Referências}

Abbad, G. da S., Loiola, E., Zerbini, T., \& Borges-Andrade, J. E. (2013). Aprendizagem em organizações e no trabalho. In: Borges, L. O., Mourão, L. \& organizadores. O trabalho e as organizações: atuações a partir da psicologia. Porto Alegre: Artmed. p. 467-527.

Borges-Andrade, J. E. (2006). Avaliação integrada e somativa em TD\&E. In: Borges-Andrade, J. E., Abbad, G. S., Mourão, L. \& organizadores. Treinamento, desenvolvimento e educação em organizações e trabalho: fundamentos para a gestão de pessoas. Porto Alegre: Artmed. p. 343-58.

Brasil. Conselho Nacional de Saúde (2012). Resolução n ${ }^{\circ}$ 466, de 12 de dezembro de 2012. Diretrizes e normas regulamentadoras de pesquisa envolvendo seres humanos. Brasília, DF: Ministério da Saúde. https://conselho.saude.gov.br/resolucoes/2012/Reso466.pdf

Brasil. Ministério da Saúde. (2015). Doenças transmissíveis no Brasil em 2014. In: Ministério da Saúde. Saúde Brasil 2014: uma análise da situação de saúde e das causas externas. Brasília: Ministério da Saúde. p. 51-157.

Brasil. Ministério da Saúde. Secretaria de Vigilância em Saúde. (2011). Tratamento Diretamente Observado (TDO) da tuberculose na atenção básica: protocolo de enfermagem. Brasília: Ministério da Saúde.

Brasil. Ministério da Saúde. Secretaria de Vigilância em Saúde. Departamento de Vigilância das Doenças Transmissíveis. (2018). Manual de Recomendações para o Controle da tuberculose no Brasil. Brasília: Ministério da Saúde. 
Research, Society and Development, v. 10, n. 13, e332101321262, 2021

(CC BY 4.0) | ISSN 2525-3409 | DOI: http://dx.doi.org/10.33448/rsd-v10i13.21262

Ceccim, R. B. (2005). Educação Permanente em Saúde: descentralização e disseminação de capacidade pedagógica na saúde. Ciênc. saúde coletiva, 10(4): 975986. http://dx.doi.org/10.1590/S1413-81232005000400020

Duarte, S. J. H., Lima, D. C., Barreira, G. C., \& Peres, W. S. (2011). A educação permanente como possibilidade no diagnóstico precoce da tuberculose. Arquivos Catarinenses de Medicina, 40(1):28-33. http://www.acm.org.br/revista/pdf/artigos/843.pdf

Lima, L. M., Harter, J., Tomberg, J. O., Vieira, D. A., Antunes, M. L., \& Cardozo-Gonzales, R. I. (2016). Avaliação do acompanhamento e desfecho de casos de tuberculose em município do sul do Brasil. Rev. Gaúcha Enferm., 37(1): e51467. https://doi.org/10.1590/1983-1447.2016.01.51467

Mariano, M. R., Brito, C. M. S., Meireles, D. S., Freitas, S. S., Aguiar, V. S., \& Neto, D. C. B. (2015). A equipe multiprofi ssional e sua qualificação junto à assistência ao paciente com tuberculose. Rev Interd., 8(4):11-18. https://revistainterdisciplinar.uninovafapi.edu.br/index.php/revinter/article/view/516

Moreira, K. S., Lima, C. A., Vieira, M. A., \& Costa, S. M. (2017). Educação permanente e qualificação profissional para atenção básica. Rev Saude e Pesquisa, 10(1):101-109. https://doi.org/10.17765/2176-9206.2017v10n1p101-109

Noe, R. A., Clarke, A. D. M., \& Klein, H. J. (2014). Learning in the twenty-first-century workplace. Ann. Rev. Organ. Psychol. Organ. Behav., 1: 245-75. https://doi.org/10.1146/annurev-orgpsych-031413-091321

Polit, D. F., Beck, C. T., \& Hungler, B. P. (2009). Fundamentos de pesquisa em enfermagem. 3ed. Porto Alegre: Artes Médicas.

Queiroga, F., Andrade, J. M., Borges-Ferreira, M. F., Nogueira, R., \& Abbad, G. S. (2012). Medidas de aprendizagem TD\&E fundamentos teóricos e metodológicos. In: Abbad, G. S., Mourão, L., Menezes, P. P., Zerbini, T., Borges-Andrade, J. E., Vilas-Boas, R., \& organizadores. Medidas de avaliação em treinamento, desenvolvimento e educação: Ferramentas para gestão de pessoas. Porto Alegre: Artmed. p. 106-24.

Reeves, S. (2016). Why we need interprofessional education to improve the delivery of safe and effective care. Interface (Botucatu), 20(56):185-197. https://doi.org/10.1590/1807-57622014.0092

Silva, L. M. C., Surniche, C. A., Sicsú, A. N., Mitano, F., Nogueira, J. A., Santos, C. B., Cunha, F. T. S., \& Palha, P. F. (2015). Elaboração e validação semântica de um instrumento de avaliação da transferência do tratamento diretamente observado como política de controle da tuberculose. Rev Panam Salud Publica, 38(2):129-35. https://www.scielosp.org/article/rpsp/2015.v38n2/129-135/\#

Tavares, R. (2010). Aprendizagem significativa, codificação dual e objetos de aprendizagem. Rev. brasileira de informática na educação, 18(2). http://dx.doi.org/10.5753/rbie.2010.18.02.04

World Health Organization. (2019). Global Tuberculosis Report 2019. Geneva: WHO.

Yost, J., Ciliska, D., \& Dobbins, M. (2014). Evaluating the impact of an intensive education workshop on evidence-informed decision-making knowledge, skills, and behaviours: a mixed methods study. BMC Medical Education, 14(13). https://doi.org/10.1186/1472-6920-14-13

Zoboli, E. L. C. P., \& Soares, F. A. C. (2012). Capacitação em bioética para profissionais da Saúde da Família do município de Santo André, SP. Rev. esc. enferm. USP, 46(5):1248-1253. https://doi.org/10.1590/S0080-62342012000500029 